\title{
Considerações biométricas do sistema respiratório de carcará (Polyborus plancus)
}

\author{
Biometric parameters of the respiratory system in the southern caracara
}

\author{
Vanessa Sobue Franzo ${ }^{{ }^{*}}$ Silvana Martinez Baraldi Artoni ${ }^{\mathrm{I}}$ Valcinir Aloísio Scalla Vulcanii ${ }^{\mathrm{II}}$ \\ Fabiana Ribeiro Barreiro ${ }^{\mathrm{III}}$ Karin Werther $^{\mathrm{IV}}$ Alex SagulaV $^{\mathrm{V}}$
}

\begin{abstract}
RESUMO
Neste trabalho, foram estudados os parâmetros biométricos das vísceras que compõem o sistema respiratório de oito carcarás de ambos os sexos, com pesos corpóreos e idades diferentes. As aves foram eutanasiadas, evisceradas e dissecadas. Em seguida os diferentes componentes do sistema respiratório foram estendidos em uma superfície plana, medidos com o auxílio de uma fita métrica em escala milimétrica e pesados em balança eletrônica de precisão. A análise estatística utilizada foi o teste $T$ de Student com o nível de significância $P \leq 0,05$. Foi observado que, em carcarás fêmeas, o comprimento da laringe foi significativamente maior do que em carcarás machos. Além disso, a traquéia de carcarás fêmeas possui um menor comprimento do que a de galinha. Também foi observado que a relação entre o peso corpóreo e o peso da traquéia e do pulmão de carcarás é maior do que em Gallus gallus domesticus.
\end{abstract}

- NOTA -
Palavras-chave: ave, carcará, sistema respiratório, anatomia, Polyborus plancus.

\section{ABSTRACT}

The biometric parameters of the internal guts that make up the respiratory system of eight Southern Caracara of both sexes, with different weights and ages were studied. The birds were sacrificed, dissected and the visceral components of the respiratory system were exposed into a plane surface. The length was measured with the support of a millimeter based measuring gauge, and the weight was obtained via electronic precision scale. For the purpose of statistical analysis the T Student test was used with a significance level of $P \leq 0.05$. The female Southern Caracara, larynx was significantly larger than in males. The trachea is small length than in chickens and that, the relationship between the corporal weight and the trachea and the lung's weight of the caracara are greater than the Gallus gallus domesticus.

Key words: bird, Southern Caracara, respiratory system, anatomy, Polyborus plancus.

Não há relatos na literatura sobre os parâmetros biométricos da traquéia, da siringe, dos brônquios primários e dos pulmões do carcará (Figura 1), visto que essa espécie avícola silvestre da família Faconidae é de extrema importância, pois ela convive com outras aves e outros animais tanto no campo, quanto nas cidades (SICK, 1988; SICK, 2001). O presente trabalho avalia as medidas biométricas do sistema respiratório de carcarás machos e fêmeas, correlacionando os dados e verificando possíveis diferenças com outras espécies aviárias, uma vez que o sistema respiratório das aves apresenta características peculiares dentre os vertebrados.

Foram utilizados oito exemplares de carcará Polyborus plancus de pesos corpóreos e faixas etárias

'Departamento de Anatomia Animal, Universidade Federal de Tocantins (UFT), Campus Araguaína, Araguaína, TO, Brasil. E-mail: vsfranzo@yahoo.com.*Autor para correspondência.

IIDepartamento de Morfologia e Fisiologia Animal, Faculdade de Ciências Agráras e Veterinárias (FCAV), Universidade Estadual Paulista (UNESP), Campus Jaboticabal, Jaboticabal, SP, Brasil.

IIIPrograma de Pós-graduação em Cirurgia Veterinária, Departamento de Clínica e Cirurgia Veterinária, FCAV, UNESP, Jaboticabal, SP, Brasil.

${ }^{\text {IV }}$ Curso de Medicina Veterinária, FCAV, UNESP, Jaboticabal, SP, Brasil..

vDepartamento de Patologia Animal, FCAV, UNESP, Jaboticabal, SP, Brasil..

${ }^{\mathrm{VI} U n i v e r s i d a d e ~ d e ~ A r a r a q u a r a ~(U N I A R A), ~ c a m p u s ~ d e ~ A r a r a q u a r a, ~ A r a r a q u a r a, ~ S P, ~ B r a s i l . ~}$ 


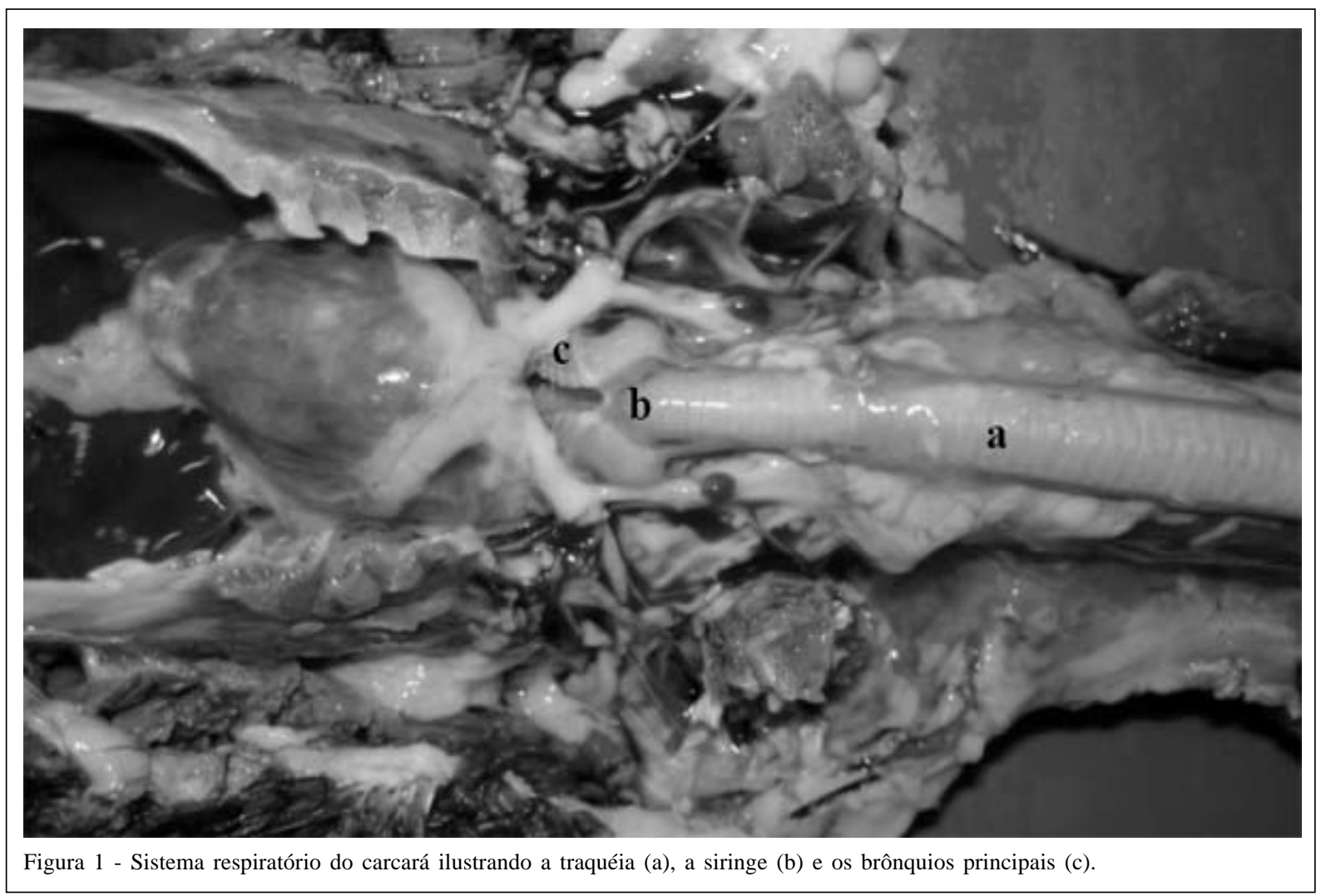

diferentes, sendo quatro machos e quatro fêmeas, provenientes do Setor de Silvestres, do Departamento de Patologia Animal, da Universidade Estadual Paulista (Unesp), campus Jaboticabal. A licença do IBAMA foi obtida por meio do processo nํㅜ 02027000933/05-47.

As aves foram anestesiadas com Zolazepam ${ }^{1}$ na dose $10 \mathrm{mg} / \mathrm{kg}$ intramuscular, no músculo peitoral superficial, e em seguida, utilizou-se cloreto de potássio na dose de $2 \mathrm{mg} \mathrm{kg}^{-1}$ de peso vivo, intravenoso, para a eutanásia do animal. Depois da abertura da cavidade torácica, o trato respiratório foi separado em blocos e foi convenientemente preparado. Sem sofrer estiramento, o trato respiratório foi colocado sobre um plano horizontal e, cada segmento foi pesado e medido, e as medidas foram devidamente anotadas. A pesagem foi realizada com o auxílio de uma balança eletrônica de precisão ${ }^{2}$, e a medida foi feita com a utilização de um paquímetro ${ }^{3}$. Os dados foram analisados estatisticamente pelo teste $\mathrm{T}$ de Student com o nível de significância $\mathrm{P} \leq 0,05$. Os termos utilizados neste trabalho estão de acordo com a Nomina anatomica avium (BAUMEL, 1993).

LUDDERS (2001) relatou que o ar entra pela boca ou pela narina, passa por meio da laringe, um órgão cartilaginoso (NEGUS, 1949), até atingir a traquéia, que se bifurca em dois brônquios primários na siringe, que é um órgão exclusivo das aves (WELTY
\& BAPTISTA, 1988) e chega aos pulmões fixos (O’CONNOR \& CLAESSENS, 2005), local em que ocorre a hematose e é responsável pela termorregulação corpórea (MACARI, et al., 2002)

Os machos obtiveram as seguintes médias de peso (Tabela 1): laringe: 0,640 $\pm 0,008$ gramas; siringe e brônquios primários: 0,872 $\pm 0,155$ gramas; traquéia 6,495 \pm 0,350 gramas; pulmão: 10,945 $\pm 1,105$ gramas. As fêmeas obtiveram as seguintes médias (Tabela 1): laringe: 0,647 $\pm 0,009$ gramas; siringe e brônquios primários: 0,987 $\pm 0,012$ gramas; traquéia: 6,687 $\pm 0,006$ gramas; pulmão 11,022 \pm 0,135 gramas.

A análise estatística demonstrou que não houve diferença significativa em relação ao peso das estruturas do sistema respiratório entre os machos e as fêmeas. No entanto, pôde se observar uma grande variação do peso dos pulmões tanto das aves machos, quanto das fêmeas, independentemente do peso corporal, de acordo com KING \& PAYNE (1960), o peso dessa estrutura é bastante variável de ave para ave. Porém, os resultados mostraram que não houve aumento do peso dos pulmões em aves de maior peso corpóreo, diferentemente do que afirmaram KING \& PAYNE (1960) ao observarem que quanto maior o peso corporal, maior o peso dos pulmões tanto em aves machos, quanto em fêmeas. Além disso, foi verificado 
Tabela 1 - Peso (g) e comprimento (cm) dos diferentes componentes do sistema respiratório de carcarás machos e fêmeas.

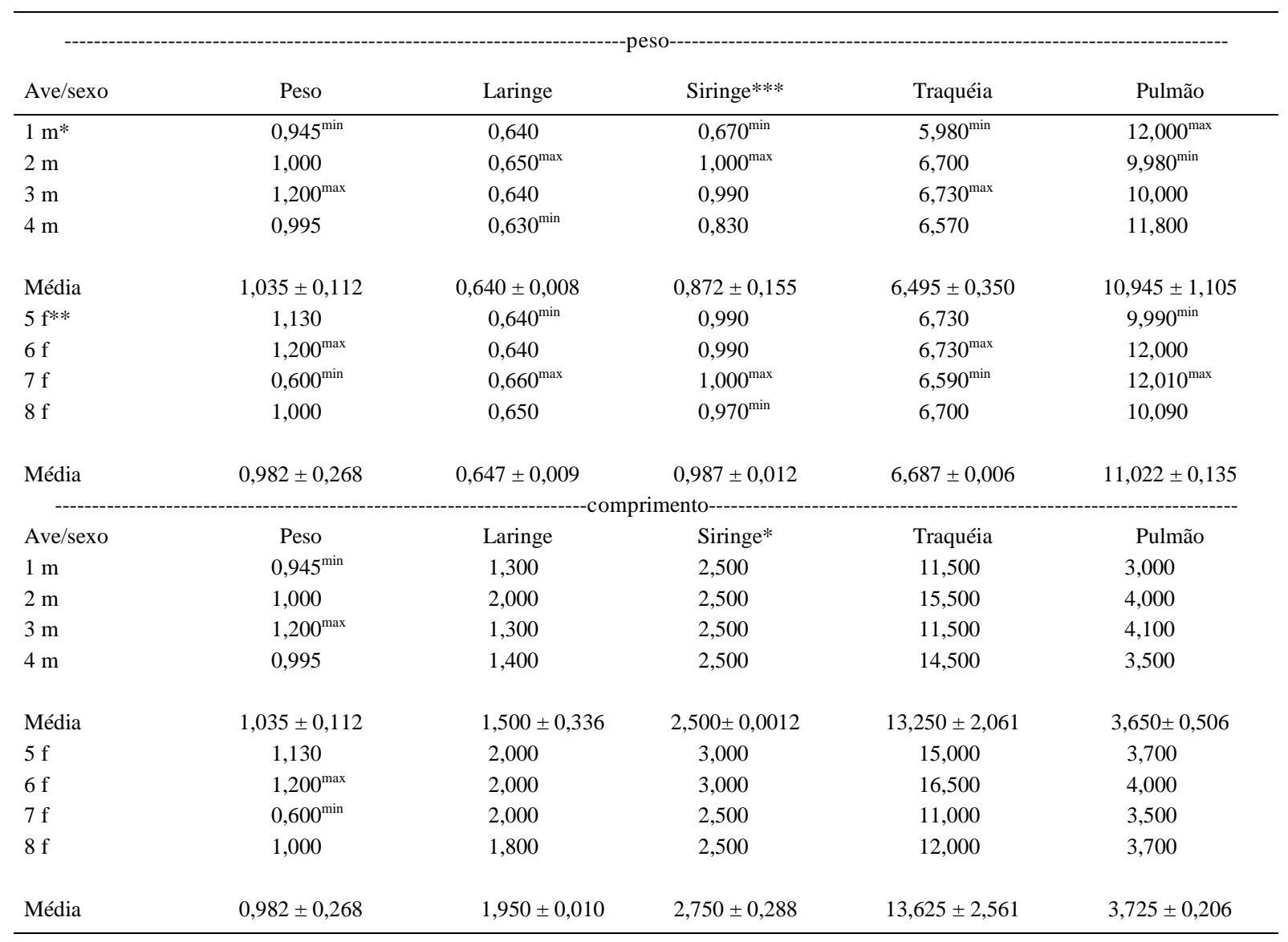

min.: valor mínimo encontrado.

max.: valor máximo encontrado.

*m: macho.

** f: fêmea.

***Corresponde ao peso da siringe mais o peso dos brônquios primários.

que, em carcarás fêmeas, o pulmão corresponde a um valor médio de $1,12 \%$ do peso corporal, enquanto que em machos, a 1,05\%. Torna-se importante salientar que não há dados sobre a idade do animal, pois eles eram oriundos da natureza, o que pode interferir nos resultados apresentados.

A relação entre o peso corpóreo e o peso da traquéia e do pulmão de carcarás é bem maior do que o encontrado por LATIMER (1924), o qual verificou que, nos machos e nas fêmeas da raça Leghorn branca de crista simples de quatro meses de idade com 1.200 gramas (g) de peso corporal, os pulmões e a traquéia pesaram cerca de oito a nove gramas, perfazendo cerca de $0,8 \%$ do total do peso corporal. Em carcarás machos, observou-se que o peso da traquéia e do pulmão é de $16,8 \%$ da média do peso corporal (103,5 gramas) nesses animais. Além disso, as aves fêmeas obtiveram 18,0\% do peso das mesmas estruturas em relação ao peso corpóreo (98,2 gramas).

Em relação ao comprimento dos diferentes órgãos que compõem o sistema respiratório, foi observado que os machos apresentaram as seguintes médias (Tabela 1): laringe: $1,500 \pm 0,336$ centímetros; siringe e brônquios primários: 2,500 $\pm 0,0012$ centímetros; traquéia: 13,250 \pm 2,061 centímetros; pulmão: 3,650 \pm 0,506 centímetros. Os dados apresentados na tabela 1 mostram que, para fêmeas, a laringe possui 1,950 $\pm 0,010$ centímetros; siringe e brônquios primários possuem $2,750 \pm 0,288$ centímetros; traquéia possui 13,625 $\pm 2,561$ centímetros e pulmão possui 3,725 $\pm 0,206$ centímetros.

A análise estatística ao nível de significância $\mathrm{P} \leq 0,05$ demonstrou que a laringe das fêmeas é maior em comprimento do que a dos machos, diferindo do que WHITE (1968) observou em galinhas, porém, são

Ciência Rural, v.39, n.1, jan-fev, 2009. 
menos extensas do que as dos patos $(2,4$ a $2,8 \mathrm{~cm})$ e gansos (3cm) estudados por WHITE (1968). A traquéia do Polyborus plancus possui, em média, 13 centímetros, sendo a traquéia menor em comprimento do que a de uma galinha doméstica estudada por McLELLAND (1989) o qual relatou que fêmeas possuem um maior comprimento de traquéia do que machos. Isso foi verificado também em carcarás, nos quais foi observado que a traquéia de fêmeas $(13,625 \pm 2,561$ centímetros) é maior do que a traquéia de machos $(13,25 \pm 2,061$ centímetros).

Em relação ao comprimento dos pulmões, foi observado que machos e fêmeas possuem pesos relativamente semelhantes, pois o pulmão de aves é fixo e possui pouca distensibilidade ( $\mathrm{O}^{\prime} \mathrm{CONNOR} \&$ CLAESSENS, 2005).

Nas condições experimentais deste trabalho, foi concluído que o sistema respiratório do carcará possui uma estrutura para propiciar a respiração e o canto da ave com órgãos semelhantes à galinha doméstica. Foi observado que o peso da traquéia e dos pulmões em relação ao peso corpóreo dos carcarás em machos e fêmeas é maior do que o de galinhas domésticas. A laringe das fêmeas é significativamente maior em comprimento do que em machos, apesar de ambos os sexos não apresentarem dimorfismo sexual em relação ao canto e a traquéia possuir um menor comprimento do que a de uma galinha.

\section{FONTES DE AQUISIÇÃO}

${ }^{1}$ Virbac do Brasil - São Paulo, SP

${ }^{2}$ Micronal - São Paulo, SP

${ }^{3}$ Starrett Indústria e Comércio Ltda - Itu, SP

\section{COMITÊ DE ÉTICA E BIOSSEGURANÇA}

O presente trabalho de pesquisa foi aprovado pela Comissão de Ética e Bem Estar Animal (CEBEA) sob o protocolo no 007590-05 e está de acordo com os Princípios Éticos na Experimentação Animal, adotado pelo Colégio Brasileiro de Experimentação (COBEA).

\section{REFERÊNCIAS}

BAUMEL, J.J. Handbook of avian anatomy: Nomina Anatomica Avium. 2.ed. Cambridge: Nuttal Ornithological Club, 1993. 779p.

KING, A.S.; PAYNE, D.C. The maximum capacities of the lungs and air sacs of Gallus gallus domesticus. Journal of Anatomy, v.96, p.495-503, 1960.

LATIMER, H.B. Post-natal growth of the body, systems and organs of the single-comb White Leghorn chicken. Journal of Agriculture Research, v.29, p.363-397, 1924.

LUDDERS, J.W. Inhaled anesthesia for birds. In: GLEED, R.D.; LUDDERS, J.W. (Eds.). Recent advances in veterinary anesthesia and analgesia: companion animals. Ithaca, NY.: International Veterinary Information Service, 2001.

MACARI, M. et al. Fisiologia aviária aplicada a frangos de corte. In: FURLAN, R.L.; MACARI, M. Termorregulação. São Paulo: FUNEP/Unesp, 2002. Cap.17, p.210-228.

McLELLAND, J. Larynx and trachea. In: KING, A.S.; MCLELLAND, J. (Eds.). Form and function in birds. London: Academic, 1989. V.4, p.69-103.

NEGUS, V.E. The comparative anatomy and physiology of the larynx. London: Willian Heinemann Medical, 1949. Chap.11, p.155-182.

O’CONNOR, P.M.; CLAESSENS, L.P. Basic avian pulmonary design and flow-through ventilation in non-avian theropod dinosaurs. Nature v.436, p.253-256, 2005.

SICK, H. Ornitologia brasileira, uma introdução. 3.ed. Brasília: Universidade de Brasília, 1988. V.01, cap.08, p.117125 .

SICK, H. Ornitologia brasileira. Família Falconidae. 3.imp. :Rio de Janeiro: Nova Fronteira, 2001. Parte 2, cap.10, p.243-269.

WELTY, J.C; BAPTISTA, L. The life of birds. 4.ed. New York: Saunders College, 1988. p.340.

WHITE, S.S. Movements of the larynx during crowing in the domestic cock. Journal of Anatomy, v.103, p.390-392, 1968. 